\title{
THE SIGNIFICANCE IN HUMAN STEREOTACTIC BRAIN SURGERY OF INDIVIDUAL VARIATION IN THE DIENCEPHALON AND GLOBUS PALLIDUS*
}

\author{
BY \\ J. B. BRIERLEY and ELISABETH BECK \\ From the Department of Neuropathology, Institute of Psychiatry, Maudsley Hospital, London
}

Access to deeply seated parts of the brain with minimal damage to structures other than the target itself was achieved in the experimental animal by Horsley and Clarke (1908). A coordinate system was employed based upon three fundamental planes, the mid-sagittal plane, a horizontal plane passing through the external auditory meati and the inferior margins of the orbits (the Frankfurt plane), and a coronal plane through the auditory meati at right angles to the latter. Thus the position of any point in the brain could be defined by three coordinates related to these three planes.

It may fairly be said that the stereotactic technique opened a new chapter in the anatomical and physiological investigation of the animal brain and a vast literature has accumulated recording the results of its application to animals of many species from rodent to primate. Nevertheless while a reasonable constancy of body weight will ensure adequate accuracy in placing the needle tip in small animals, the factor of individual variation becomes more important in larger brains. Thus for the cat, Loeu enfeld and Altman (1956) have demonstrated the lack of a constant relationship between brain structures and salient landmarks in the skull. Again, in the monkey, skull shapes may vary considerably betw een animals of similar body weight (Olszewski, 1951).

The first application of the stereotactic principle to the human brain was made by Spiegel, Wycis, Marks, and Lee (1947) in an attempt to replace the operation of prefrontal leucotomy by interruption of thalamocortical pathways in the thalamus itself. It was recognized that coordinates of subcortical structures could never be estimated with any degree of accuracy if based upon bony landmarks alone. Further there was ".... great variability in the relation of the inter-aural plane to an intracerebral reference point, for example, the centre of the pineal

*A preliminary communication has already been presented (Brierley and Beck, 1957). body or of the posterior commissure" (Spiegel and Wycis, 1952).

The chapter on variability studies forms a valuable part of this atlas and while indicating the value of the technique for targets as large as the dorsomedial nucleus of the thalamus or the globus pallidus, it also provided an incentive to a search for a method that might assure greater accuracy and thereby justify operations upon the smaller diencephalic nuclei.

An important step forward consisted in the selection of a horizontal plane independent of any bony landmark and passing through midline diencephalic structures identifiable in a lateral radiograph in which the third ventricle was filled with air or a radio-opaque material. Several such horizontal planes have been used, the majority passing through the posterior commissure posteriorly, and through either the lower margin of the interventricular foramen (Hassler and Riechert, 1954) or the upper border of the anterior commissure (Talairach, de Ajuriaguerra, and David, 1952) anteriorly. The distance between these anterior and posterior points is measured in the patient's encephalogram (with correction for $x$-ray magnification) and in the "atlas" brain. The ratio between them has been used (e.g., Hassler and Riechert, 1954) as a factor by which the antero-posterior coordinate of a particular nucleus in the "atlas" brain can be corrected for that of the patient. The important assumption is therefore made that these two points, but particularly the posterior commissure, maintain a constant spatial relationship to thalamic and other diencephalic structures.

The primary purpose of the present investigation was to test this assumption in a large series of human brains (Part I). From the outset it became apparent that the thalamic nuclear pattern at posterior commissure level varied considerably from case to case. An attempt was therefore made to define a more reliable landmark from which to measure the anteroposterior coordinates of thalamic nuclei (Part II). 
The data emerging from both studies cannot be regarded as any form of stereotactic atlas of the diencephalon; they indicate solely individual variation and relationships and not the dimensions of individual nuclei.

Additional information has also been obtained concerning the variation in the position of the globus pallidus.

\section{MATERIAL AND METHODS}

In all, 53 hemispheres from 47 formalin-fixed and suspended brains collected from general hospitals were used in the investigation. Any brain showing the least degree of distortion (particularly of the brain-stem) was rejected. However, a number showing slight to moderate cortical atrophy or a slight to moderate degree of hydrocephalus (features likely to be present in a proportion of patients selected for stereotactic operations) were included. The weight range was 1,170 to $1,615 \mathrm{~g}$.

Each brain was divided in the mid-sagittal plane. A " $O$ " horizontal plane connecting the lower border of the interventricular foramen and the nearest point on the posterior commissure was marked with a fine needle on the medial aspect of the thalamus. This line was extended forwards across the septum pellucidum or rostrum of the corpus callosum and backwards across the midbrain tectum.

In each hemisphere the distance, V (Fig. 1a), between posterior commissure and interventricular foramen was measured and also the "total thalamic length", $T$, from the interventricular foramen to the point where the " $O$ " horizontal plane intersects the posterior surface of the pulvinar.

In each of the $\mathbf{4 0}$ hemispheres used in the first part of this study a coronal cut was made at right angles to the horizontal plane at the level of the anterior edge of the posterior commissure. An Edinger macrotome was used to ensure that this first cut passed precisely at right angles to the mid-sagittal plane. Further parallel cuts were then placed at 3 to $5 \mathrm{~mm}$. intervals, the slice thickness being adjusted so that one cut passed through the mid-thalamic point, i.e., half way between the posterior commissure and the interventricular foramen, and one through the lower border of the interventricular foramen (Fig. 1a).

Histological examination was carried out on the slice adjacent to the mid-thalamic and posterior commissure planes. The " $O$ " horizontal plane was marked on the coronal face of each by two small punch holes aligned at right angles to the sagittal plane at the level of the "O" plane itself. From each slice a block was cut to include thalamus, sub-thalamus, and a variable portion of the pallidum. Frozen sections were cut at $50 \mu$ and stained for nerve cells (Nissl's method) and for myelin sheaths (Woelke's modification of Weigert Pal). In view of the rapid change in thalamic nuclear configuration, especially at the posterior commissure, care was taken to stain the first two sections from each block.

Projection diagrams were drawn from the sections and the "O" horizontal plane was indicated by a line passing through the two small punch holes referred to above.

In the remaining 13 hemispheres (Part II) the "O" hori- zontal plane was marked as before. The "total thalamic length" was then measured and coronal cuts were placed at $0,25,50,75$, and $100 \%$ of this value measured forwards from the pulvinar thus producing four slices of equal thickness. Projection diagrams were drawn to illustrate all but the zero percentage level; these included basal ganglia as well as thalamic structures. Myelinstained sections of the thalamus were prepared for the 25 and $50 \%$ levels only and supplemented by Nisslstained sections to define nuclear configuration where necessary. At the 75 and $100 \%$ levels, all structures were macroscopically visible so that the diagrams could be prepared from the slices themselves.

For the globus pallidus, the position of its lateral and inferior borders with respect to the midline and horizontal zero planes was determined at the 100,75 , and $50 \%$ levels, the medial tip at the 75 and $50 \%$ planes only. It was thus possible to compare the thalamic nuclear pattern and the position of the globus pallidus, at each percentage level in all 13 hemispheres.

\section{RESULTS IN PART I \\ Relations of the Posterior Commissure (40 Hemispheres)}

Antero-posterior.-The distance between the interventricular foramen and the posterior commissure (ventricular length, V) varied from 20.0 to $26.0 \mathrm{~mm}$. (average $23.4 \mathrm{~mm}$.). There was virtually no correlation between $\mathrm{V}$ and brain weight.

The total thalamic length (T) was found to vary between 31.0 and $38.0 \mathrm{~mm}$. (average $34.5 \mathrm{~mm}$.). There was some broad correlation between total thalamic length and brain weight, so that for any particular weight, the length $\mathrm{T}$ might vary up to $\pm 2.5 \mathrm{~mm}$.

The inclination of the horizontal plane as seen on the medial surface of the hemisphere was found to be very variable. When prolonged anteriorly this line could pass anywhere between two points $1.5 \mathrm{~mm}$. below and $7.0 \mathrm{~mm}$. above the posterior end of the lamina rostralis of the corpus callosum.

The distance from the posterior commissure to the tip of the pulvinar (P) equals 26 to $41 \%$ of the total thalamic length and this value (referred to in the following as the "percentage value") had no relation to brain weight or to the total thalamic length itself. The difference between these two extreme percentage values was $15.4 \%$. This implies that in a brain of average total thalamic length $(34.5 \mathrm{~mm}$.), the posterior commissure would lie anywhere within an antero-posterior distance of $5.3 \mathrm{~mm}$.

Between the extreme positions of the posterior commissure the $\mathbf{4 0}$ brains could be grouped according to their percentage values as follows:-

(1) 25 to $29 \%$ in 12 specimens

(2) 30 to $34 \%$ in 15 specimens

(3) 35 to $39 \%$ in 10 specimens ( + three at 40,40 , and $41 \%$ ) 
Fig. 1.-Diagram of the mid-sagittal plane indicating the horizontal plane (AC) through the lower border of the interventricular foramen and anterior curvature of the posterior commissure; extended posteriorly to intersect the pulvinar at (D) and anteriorly across the septum.

$T=$ total thalamic length $(\mathrm{AD})$

$\mathrm{V}=$ ventricular length (AC)

$\mathbf{M}=$ half ventricular length $(\mathrm{AB}, \mathrm{BC})$

$\mathbf{P}=$ length of pulvinar behind posterior commissure (CD).

B. Diagram of coronal section at midthalamic level to illustrate the vertical extent $(X)$ below, and (Y) above the zero horizontal line.

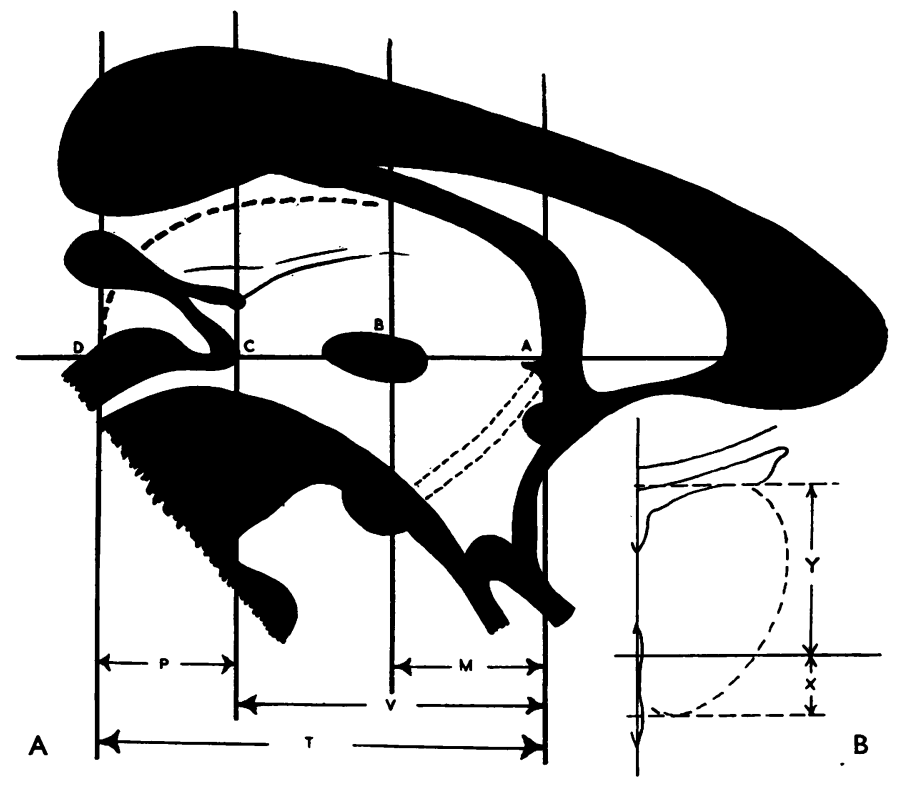

25 to $29 \%$ Level.-Within this group, the more posterior nuclear pattern comprised the pulvinar and the two geniculate bodies (Fig. 2b). The more anterior pattern included, in addition, the extreme posterior tips of the centromedian nucleus and of the nucleus ventralis posterior. The geniculate bodies might be absent or only one, the lateral, might be present.

There was one exception in this group with a percentage value of 29 in which the centromedian nucleus was fully developed and the dorsomedial nucleus was also present. It thus approximated to the features of the next group.

30 to $34 \%$ Level.-Here the nuclear pattern was more uniform (Fig. 6b) and comprised the junction of the pulvinar with the dorsomedial nucleus, the nucleus lateralis posterior, and the full development of the centromedian, ventralis posteromedialis, and ventralis posterolateralis nuclei. The extreme anterior tip of the lateral geniculate body was present in five specimens.

35 to $41 \%$ Level.-The nuclear configuration differed from that in the previous group in that the dorsomedial nucleus was fully developed. The nucleus lateralis dorsalis was present in seven specimens. In all but two, the lateral geniculate body was no longer seen (Fig. 2a).

Left-right Differences.-Nine whole brains were available in which to compare left and right sides.

In all nine the left and right ventricular lengths did not differ by more than $1 \mathrm{~mm}$. In each of six brains in which the total thalamic lengths and thus the percentage values of the posterior commissure were very similar, there was no appreciable difference between the nuclear patterns at the level of the latter.

In three brains (Fig. 3) with identical ventricular lengths there were left-right differences in total thalamic length (up to $3.5 \mathrm{~mm}$.) and thus different percentage values and nuclear configurations at the posterior commissure level. The larger percentage values exhibited the more anterior characteristics.

Vertical.-In the vertical plane the position of the posterior commissure (and therefore that of the "O" horizontal plane) with respect to the thalamic nuclei was also subject to variation. The distance from the " $O$ " horizontal plane to the lower (X) and the upper (Y) border of the thalamus (Fig. 1b) were measured at the posterior commissure level in the three groups of brains described above. The ratio $\mathrm{Y} / \mathrm{X}$ was recorded and also the total thalamic height $(X+Y)$.

\begin{tabular}{|c|c|c|c|c|}
\hline & $\mathbf{x}$ & $\mathbf{Y}$ & $\mathbf{X}+\mathbf{Y}$ & $\mathbf{Y} / \mathbf{X}$ \\
\hline $\begin{array}{l}\text { Group } 1 \\
\text { Group } 2 \\
\text { Group } 3\end{array}$ & $\begin{array}{l}0-4 \mathrm{~mm} . \\
1-4 \mathrm{~mm} . \\
1-5 \mathrm{~mm} .\end{array}$ & $\begin{array}{l}12.0-16.5 \\
13.0-17.5 \\
11.5-18.0\end{array}$ & $\begin{array}{l}12.5-17 \cdot 75 \\
15.0-20.5 \\
13.5-19.5\end{array}$ & $\begin{array}{l}3 \cdot 2-33 \cdot 0 \\
3 \cdot 2-12 \cdot 0 \\
2 \cdot 6-14 \cdot 2\end{array}$ \\
\hline
\end{tabular}

\section{Relations of the Mid-thalamic Point}

The position of the mid-point of the line between the interventricular foramen and the posterior commissure varied between 60.5 and $70 \%$ of the total thalamic length measured from the pulvinar. 


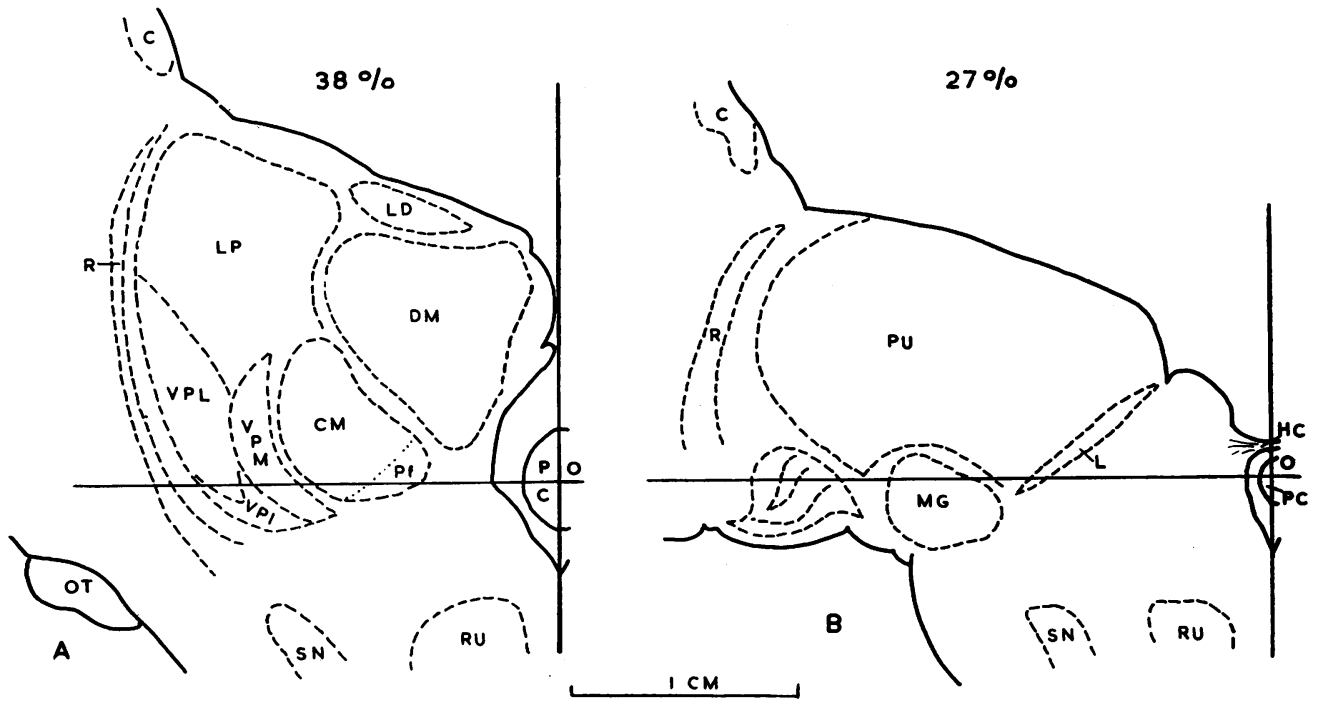

Fig. 2.-Disparity between the thalamic nuclear configuration at the posterior commissure level in two hemispheres with percentage values (of posterior commissure) of (A) 38 and of (B) 27.

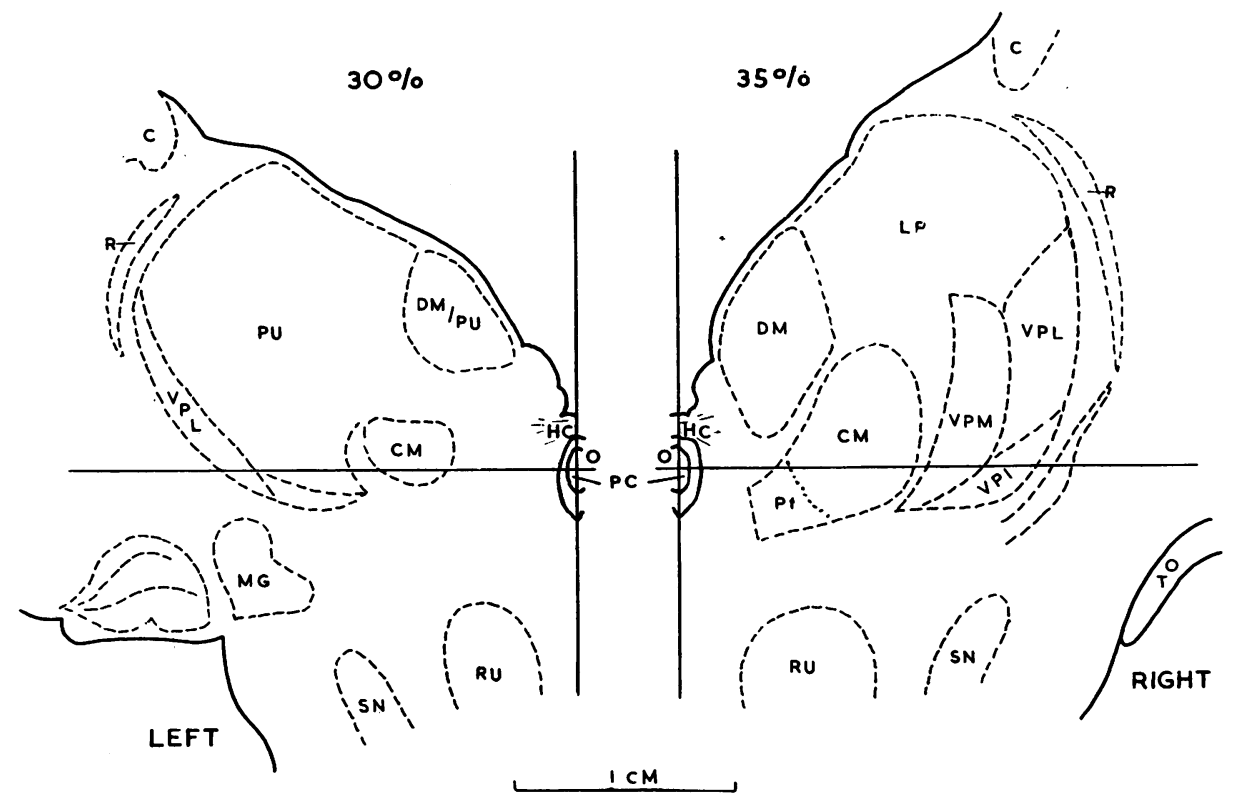

FIG. 3.-Disparity between the thalamic nuclear configuration at posterior commissure level in one brain. The ventricular length is equal $(24 \mathrm{~mm}$.) but the total thalamic length is unequal (left $30 \mathrm{~mm}$., right $37 \mathrm{~mm}$.).

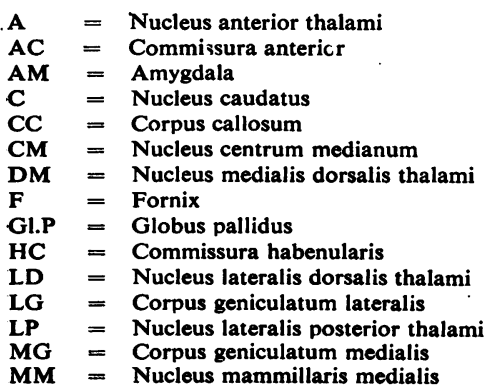

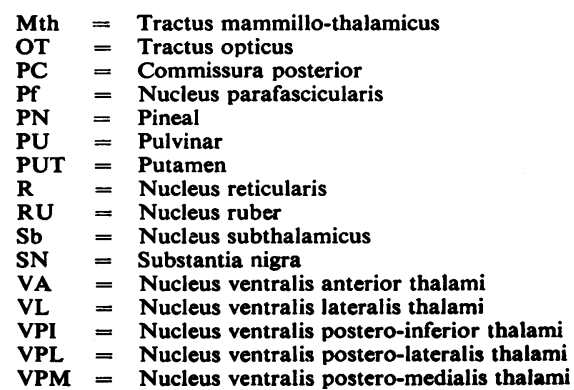



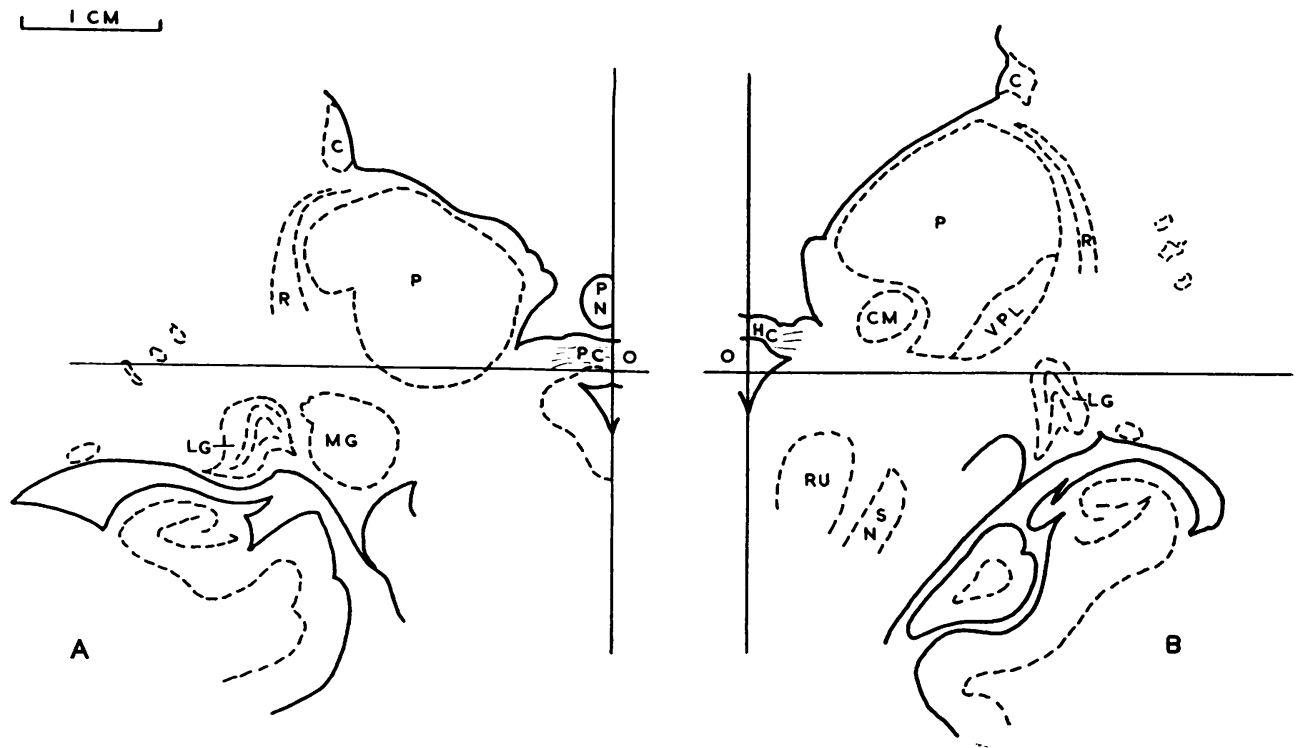

$25 \%$
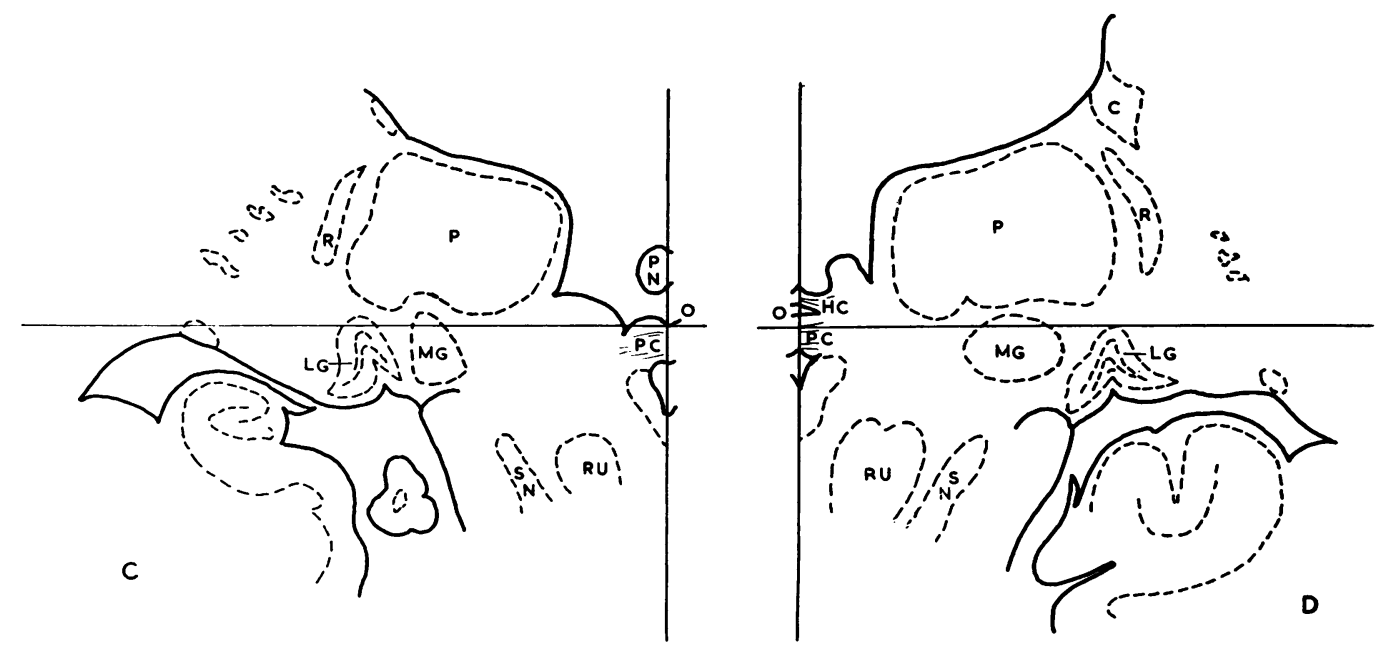

Fig. 4.- Typical and closely similar thalamic nuclear configuration at $25 \%$ of the total thalamic length measured from the pulvinar. The percentage values of the anterior curvature of the posterior commissure were $A=40, B=23, C=36, D=28$.

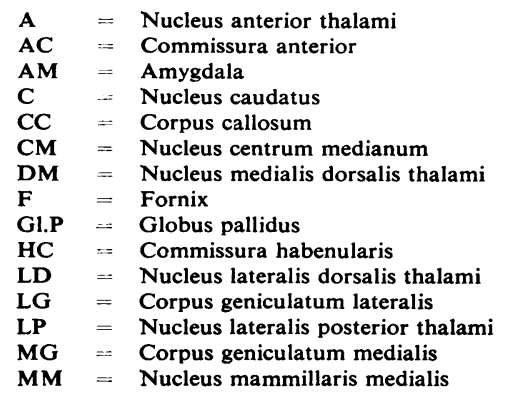

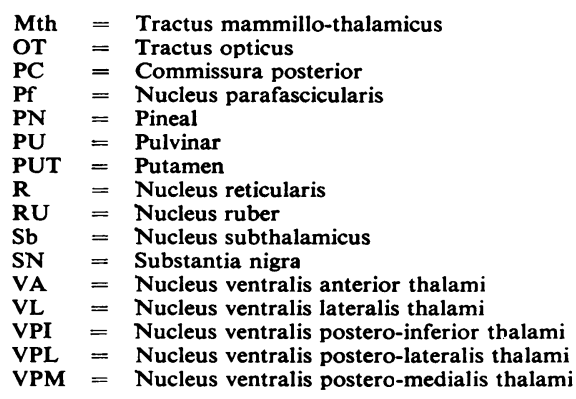


This represented a distance of about $3.3 \mathrm{~mm}$. in a brain of average total thalamic length $(34.5 \mathrm{~mm}$.). The variation in nuclear pattern between the two extremes was not great. The dorsomedial and ventrolateral nuclei were seen in all cases, while at the anterior limit of variation the posterior end of the anterior complex was seen. At the posterior limit, the nucleus lateralis dorsalis was present and the anterior complex was absent.

In the vertical plane " $X$ " varied from 0 to $5.5 \mathrm{~mm}$. and in two cases the lower border of the thalamus lay $2.0 \mathrm{~mm}$. above the " $O$ " horizontal plane. The distance " $Y$ " varied from 11.0 to $17.5 \mathrm{~mm}$., total thalamic height $(X+Y)$ from 14.0 to $21.0 \mathrm{~mm}$. and the ratio $\mathrm{Y} / \mathrm{X}$ from 2.3 to 12.4 , indicating the inconstancy of the level at which the " $O$ " horizontal plane intersects the thalamus.

It was noted that a broad relationship was demonstrable when the total thalamic height $(X+Y)$ was plotted against brain weight. The range of $(X+Y)$ for any one brain weight was $\pm 2.0 \mathrm{~mm}$.

\section{RESULTS IN PART II}

\section{Antero-posterior Organization in the Thalamus}

In this series of 13 hemispheres the distance " $V$ " varied from 21 to $26 \mathrm{~mm}$. and the total thalamic length " $T$ " from 30.5 to $38.0 \mathrm{~mm}$. The distance from the posterior commissure to the tip of the pulvinar equals 23 to $40 \%$ of the total thalamic length. In all but one case $(23 \%)$ the posterior commissure lay a variable distance in front of the $25 \%$ level.

$25 \%$ Level (Fig. 4). - The pulvinar was seen in all 13 hemispheres, the full development of both geniculate bodies appeared in nine, the lateral geniculate in three, and neither in one. The extreme posterior tip of the centromedian nucleus appeared in four specimens and the posterior end of the ventralis posterolateralis and posteromedialis in three.

At the anterior limit of variation the posterior ends of VPM, VPL, and the centromedian were present (Fig. 4B), whereas the pulvinar alone was seen at the posterior limit (Figs. 4A, C, D). The distance between these two extremes would be about $1.5 \mathrm{~mm}$. in an average total thalamic length of $34.5 \mathrm{~mm}$.

In all but one case a variable portion of the posterior commissure and periaqueductal grey matter were displayed at this level.

In the vertical plane " $X$ " varied from 0 to $3.0 \mathrm{~mm}$. In five cases the lower border of the thalamus lay up to $3.0 \mathrm{~mm}$. above the " $O$ " horizontal plane. " $Y$ " varied from 13 to $19 \mathrm{~mm}$., the total thalamic height from 12 to $18.5 \mathrm{~mm}$., and the ratio $\mathrm{Y} / \mathrm{X}$ from 4 to $12 \cdot 5$.
$50 \%$ Level (Fig. 5). - At this level the anterior tips of VPM, VPL, and the centromedian nucleus were seen. The fully developed dorsomedial nucleus lay medially, the lateralis posterior laterally, and the lateralis dorsalis dorsally. Antero-posterior variation at this level involved only a narrow band at the anterior end of VPM, VPL, and CM representing a distance of about $1.5 \mathrm{~mm}$.

Globus Pallidus.-The cross sectional areas and configuration were closely comparable throughout. The medial edge of this structure lay between 13 and $24 \mathrm{~mm}$. and the lateral border between 24 and $29 \mathrm{~mm}$. from the midline. The lower border lay from 2 to $9 \mathrm{~mm}$. below the horizontal zero plane.

In the hypothalamic region the $50 \%$ coronal plane passed through the posterior parts of the mammillary bodies in three brains and lay posterior to them in the remaining ten.

In the vertical plane " $X$ " varied from 0 to $4.5 \mathrm{~mm}$., "Y" from 15 to $21 \mathrm{~mm}$., $(X+Y)$ from 17 to $22 \mathrm{~mm}$., and the ratio $Y / X$ from 4 to 23 .

$75 \%$ Level (Fig. 5). - At this level the ventralis lateralis, the ventralis anterior, and the anterior part of the dorsomedial nuclei were seen. The anterior complex was fully developed in all cases. The extent of antero-posterior variation in the thalamus at this level was very small and probably less than a millimetre. The mammillo-thalamic tract (bundle of Vicq d'Azyr) appeared in 11 brains.

Globus Pallidus.-The cross sectional areas and internal configuration were again closely similar at this level. The medial edge lay between 12 and $19 ?$ $\mathrm{mm}$. and the lateral edge between 22 and $32 \mathrm{~mm}$. from the midline. The lower border lay from 2 to $9 \mathrm{~mm}$. below the horizontal zero plane.

Ventrally, the $75 \%$ coronal plane passed through the middle of the mammillary bodies in the two brains, through their anterior portions in two, and through the tuberal region in nine.

In the vertical plane " $X$ " varied from 0 to $5.0 \mathrm{~mm}$. and in three instances lay up to $1 \mathrm{~mm}$. above the "O" horizontal plane. "Y" varied from 12 to 20 mm., total thalamic height $(X+Y)$ from 15.5 to $21.0 \mathrm{~mm}$., and the ratio $\mathrm{Y} / \mathrm{X}$ from 2.5 to 10 .

$100 \%$ Level (Fig. 5).-This lay anterior to the thalamus and presented only the internal capsule (anterior limb) and the anterior portion of the corpus striatum.

Globus Pallidus.-Only the outer segment was seen at this level in all cases. Its lateral border lay between 15 and $23 \mathrm{~mm}$. from the midline and its lower border between 4 and $10 \mathrm{~mm}$. below the " $O$ " horizontal plane. The medial edge of the pallidum was too ill-defined macroscopically to permit of measurement. 

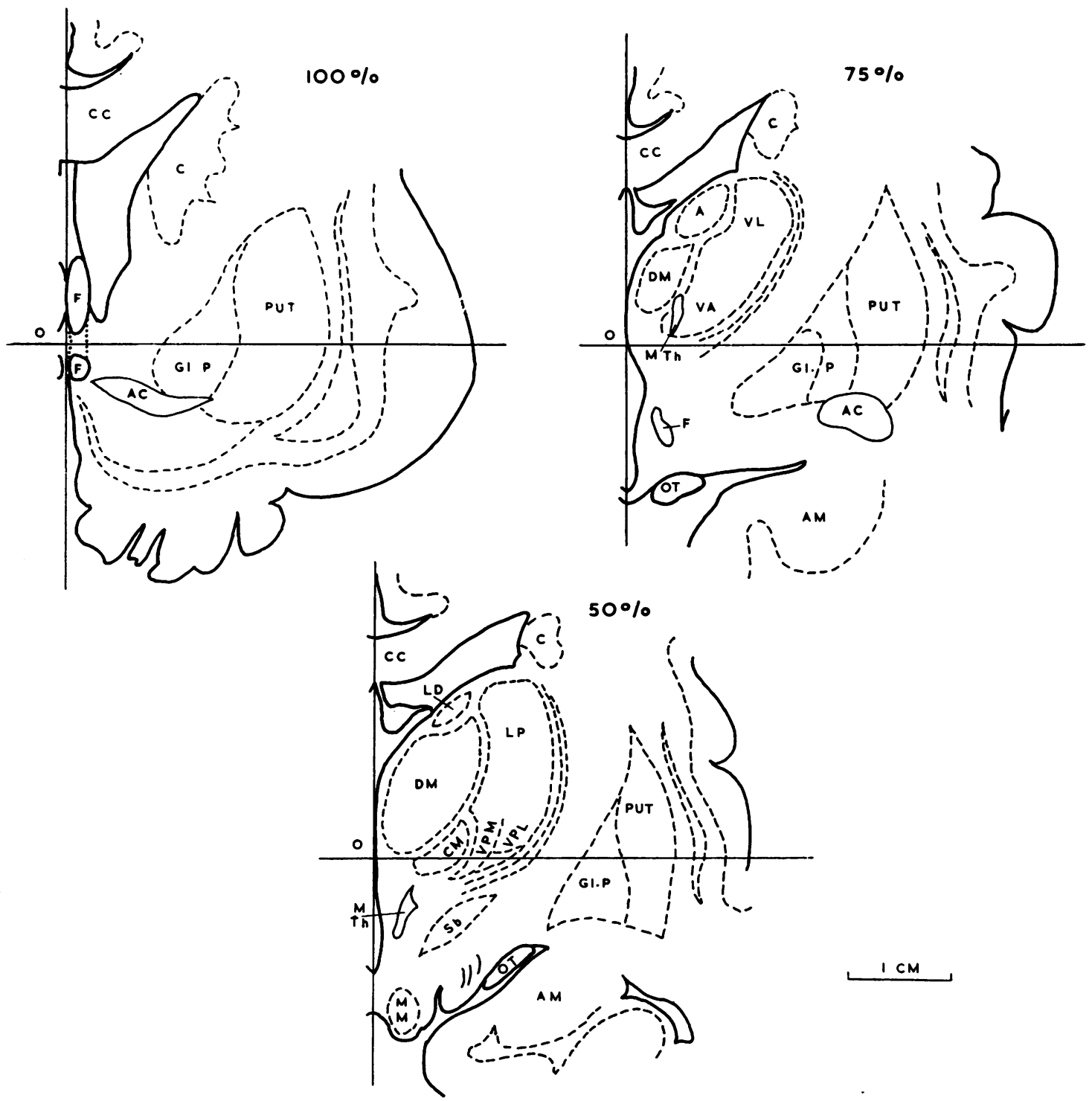

Fig. 5.-Typical nuclear configuration at 100.75 , and $50 \%$ levels of the total thalamic length measured from the pulvinar.

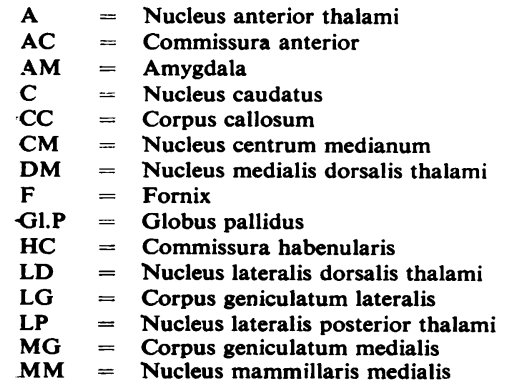

Mth = Tractus mammillo-thalamicus
OT $=$ Tractus opticus
PC $=$ Commissura posterior
Pf $=$ Nucleus parafascicularis
PN $=$ Pineal
PU $=$ Pulvinar
PUT $=$ Putamen
R $=$ Nucleus reticularis
RU $=$ Nucleus ruber
Sb $=$ Nucleus subthalamicus
SN $=$ Substantia nigra
VA $=$ Nucleus ventralis anterior thalami
VL $=$ Nucleus ventralis lateralis thalami
VPI $=$ Nucleus ventralis postero-inferior thalami
VPL $=$ Nucleus ventralis postero-lateralis thalami
VPM $=$ Nucleus ventralis postero-medialis thalami




\section{DISCUSSION}

It should again be stressed that this investigation was undertaken not to provide a stereotactic reference atlas but solely to assess the importance of individual variation in so far as it concerns spatial relationships in the diencephalon and globus pallidus.

In any study of spatial relationships in the brain, consideration of post-mortem distortion is of primary importance. In the human brain this factor is unavoidable since, in the absence of the support and volume of the pulsating vascular tree, cerebral dimensions will be reduced to an unknown and variable extent (Mark and Yakovlev, 1955). Further, the cerebrospinal fluid, in which the brain almost floats in vivo, disappears rapidly after death causing the brain to sink upon the subjacent cranium with consequent distortion. Perfusion in situ as employed by Spiegel and Wycis (1952) and by Talairach, David, Tournoux, Corredor, and Kvasina (1957) will not correct this factor as evidenced by the unequal spacing of the initially parallel guide needles inserted into the fresh specimen by the latter authors. Thus there appeared to be no greater objection to making use of brains removed as soon as possible after death and suspended (by the basilar artery) in a large volume of fixative. In such specimens furthermore, the range of thalamic length $(20.0$ to $26.0 \mathrm{~mm}$.) is similar to that encountered in a series of 14 normal encephalograms corrected for $x$-ray magnification $(22.5$ to $26.0 \mathrm{~mm}$.).

Again, the variation in the position of the posterior commissure, together with the existence of a proportionate arrangement of the thalamic nuclei along the total thalamic length suggest that neither of these findings could be attributed to distortion consequent upon fixation. Individual variation is therefore the most probable explanation of the observed differences in both the antero-posterior and the vertical relations of the posterior commissure.

In the antero-posterior axis, the posterior commissure lay between 26 and $41 \%$ (first series of 40 brains) and 23 and $40 \%$ (second series of 13 brains) of the total thalamic length measured from the tip of the pulvinar. The underlying thalamic nuclear pattern was seen to become more posterior in character the further caudalwards the posterior commissure lay along the thalamic axis (Figs. 2 and 6). Cases with identical "ventricular lengths" (22 $\mathrm{mm}$.) but different percentage values for the posterior commissure (29 to 38 ) showed correspondingly different nuclear patterns at this level (Fig. 6). On the other hand, cases with similar percentage values for the posterior commissure (36 to 37 ) and different ventricular lengths $(20.5$ to $24.0 \mathrm{~mm}$.) presented almost identical nuclear patterns (Fig. 7).
Spiegel and Wycis had already noted variability in the position of the posterior commissure, its distance from the posterior border of the lateral geniculate body or the pulvinar varying by up to $3.0 \mathrm{~mm}$. Similarly Mark and Yakovlev found that the distance from the commissure to the posterior tip of the "posteromedial portion of the ventral nucleus" varied from 2.1 to $7.2 \mathrm{~mm}$. in a series of five hemispheres. The finding of Talairach et al. that the posterior edge of the centromedian nucleus always lay at the level of the posterior commissure was not confirmed in the present material.

The existence of left-right asymmetry in the position of diencephalic nuclei was described by Delmas and Pertuiset (1954) but was not observed by Talairach et al. In the present investigation there were three brains in each of which the nuclear configuration at the posterior commissure differed considerably between the two sides. While in each brain the ventricular lengths were closely similar, the total thalamic lengths and therefore the percentage values of the posterior commissure differed. The more posterior nuclear configuration was to be seen on the side with the lower percentage value (Fig. 3).

The additional variability of the posterior commissure in the vertical plane follows from the demonstration that the zero horizontal plane through the commissure intersects the thalamus at an inconstant level. Thus in the first series, the ratio $X / Y$ varied from 2.6 to 33.0 at the coronal level of the commissure and from 2.3 to 12.4 at mid-thalamic level. Similar variation in this ratio was noted at each of the four coronal planes studied in the second series.

The practical implication of these findings is that the posterior commissure should not be used as a landmark from which to measure the anteroposterior coordinates of nuclei in the thalamus. This consideration will be of particular importance for such small structures as the sensory relay nuclei (VPL, VPM), the centromedian, and the subthalamic nuclei, where the proximity to the internal capsule makes maximum accuracy desirable.

For the antero-posterior coordinate it seems that greater accuracy could be attained by making use of the fact that the thalamic nuclei appear to occupy relatively constant proportions of the total thalamic length as suggested by the findings in the second series. The total thalamic length can be estimated in an air encephalogram in which the line from the anterior commissure or interventricular foramen to the posterior commissure is prolonged backwards to intersect the surface of the pulvinar as visualized with air in the cisterna ambiens. The anteroposterior correction factor would then be the ratio between this length in the patient's brain (corrected 


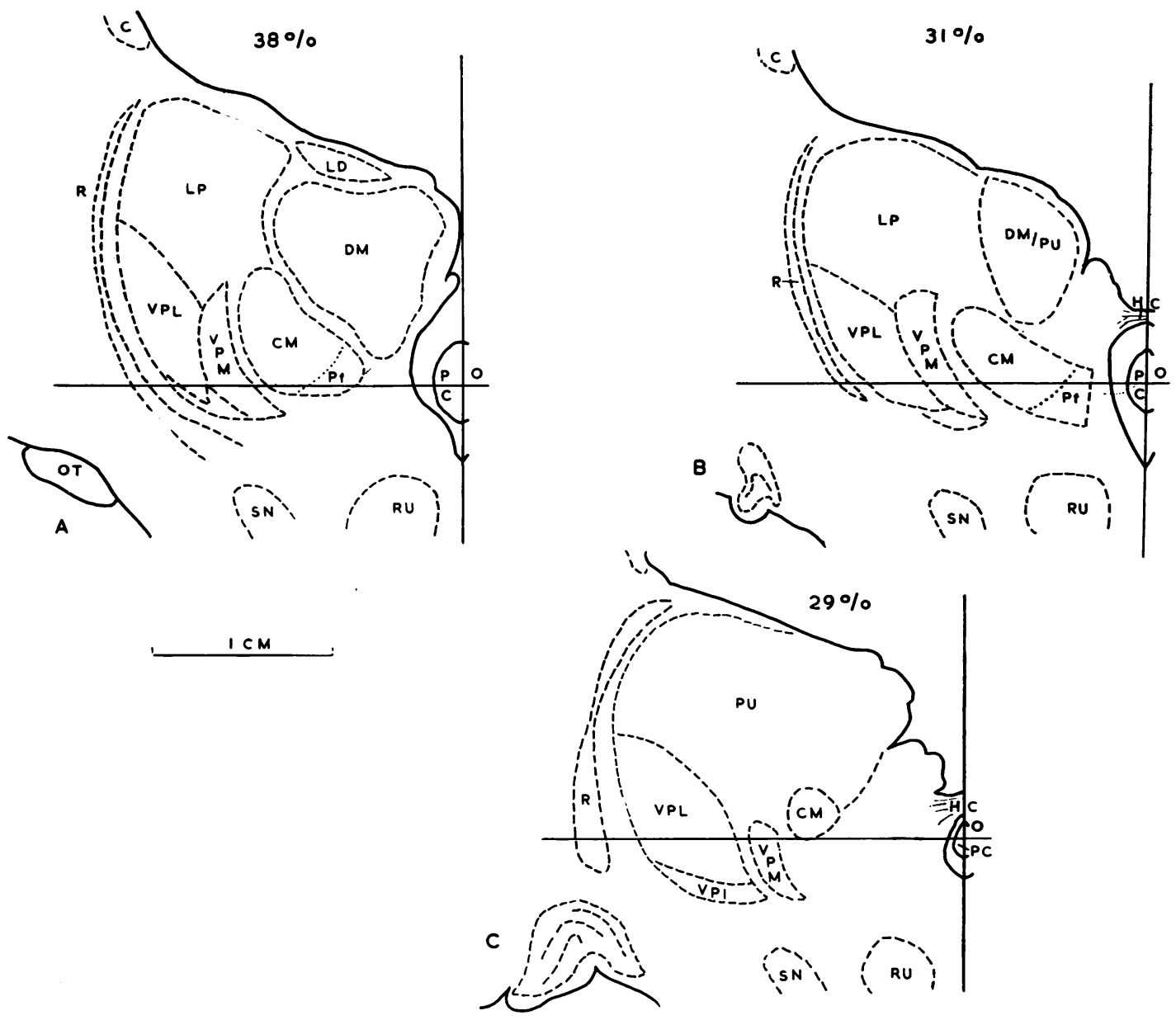

Fig. 6.-Disparity between the thalamic nuclear configuration at the posterior commissure level in three hemispheres of identical ventricular length $(22 \mathrm{~mm}$.) but different percentage values $(38,31$, and 29$)$.

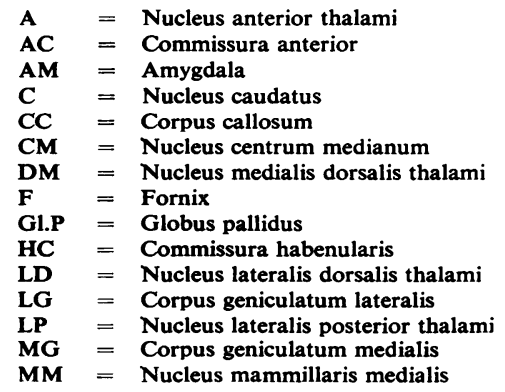



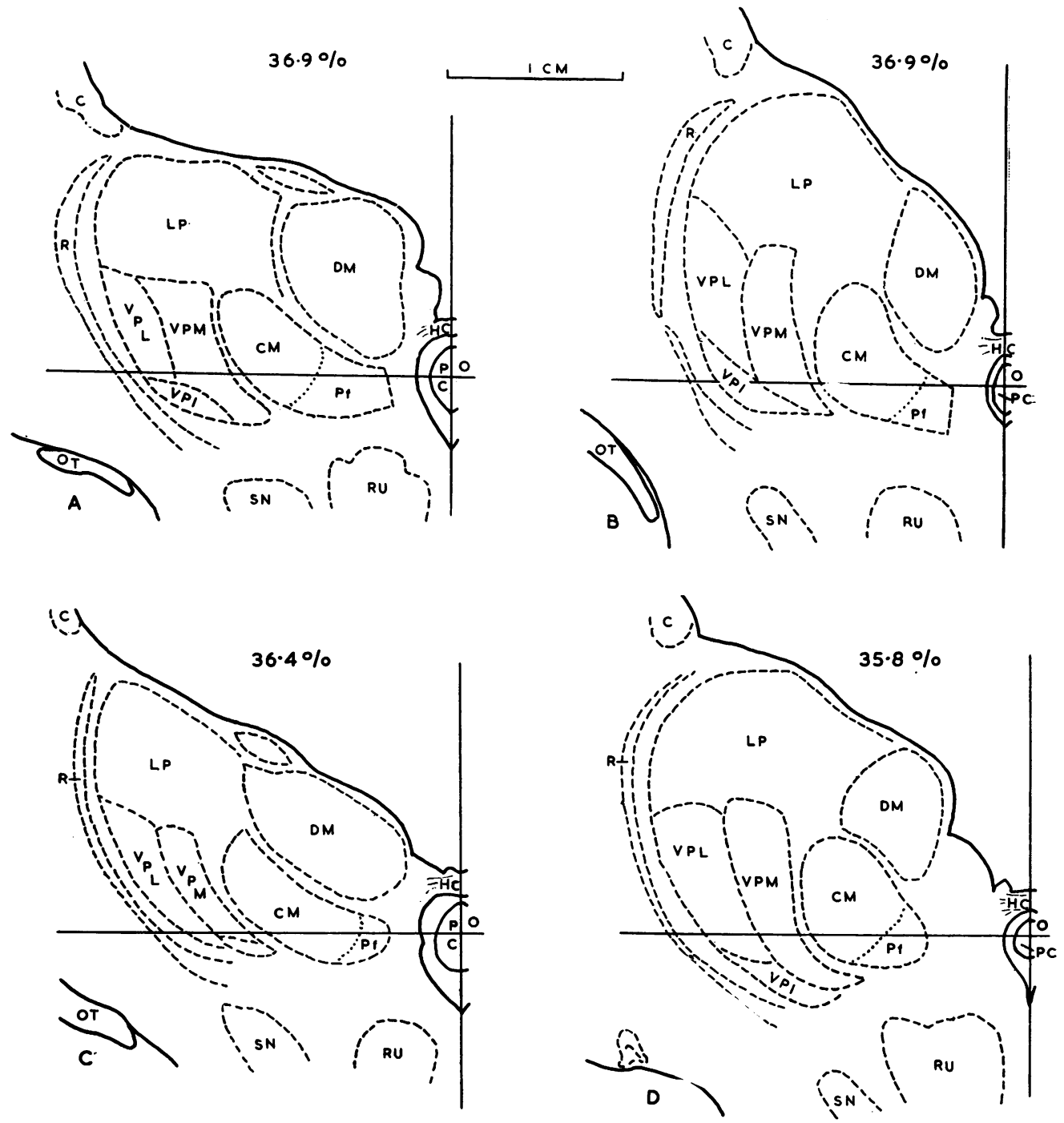

FIG. 7.-Similar thalamic nuclear configuration at the posterior commissure level in four hemispheres with closely similar percentage values ( 36 to 37 ) but different ventricular length (20 to 24$)$.

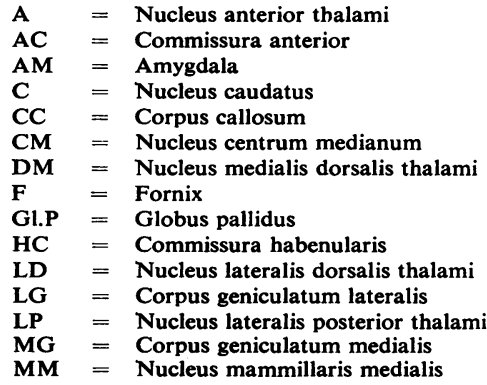

A $=$ Nucleus anterior thalami

C $=$ Nucleus caudatus

$=$ Corpus callosum

DM = Nucleus medialis dorsalis thalami

GlP $=$ Globus pallidus

HC $=$ Commissura habenularis

= Nucleus lateralis dorsalis thalami

$=$ Nucleus lateralis posterior thalam

MM = Nucleus mammillaris medialis

Mth = Tractus mammillo-thalamicus
OT $=$ Tractus opticus
PC $=$ Commissura posterior
Pf $=$ Nucleus parafascicularis
PN $=$ Pineal
PU $=$ Pulvinar
PUT $=$ Putamen
R $=$ Nucleus reticularis
RU $=$ Nucleus ruber
Sb $=$ Nucleus subthalamicus
SN $=$ Substantia nigra
VA $=$ Nucleus ventralis anterior thalami
VL $=$ Nucleus ventralis lateralis thalami
VPI $=$ Nucleus ventralis postero-inferior thalami
VPL $=$ Nucleus ventralis postero-lateralis thalami
VPM = Nucleus ventralis postero-medialis thalami


for $x$-ray magnification) and the same distance in the brain from which the stereotactic atlas had been prepared. Such an atlas should also indicate the proportion of the total thalamic length occupied by the target nucleus.

For the vertical coordinate, however, it is clear that the only valid correction factor would be one relating the total thalamic heights in the atlas and patients' brains. It is clearly impossible, however, to know the position of the lower border of the thalamus and thus its total height. In the absence of information concerning the extent of the patient's thalamus below the horizontal plane $(\mathrm{X})$, a ratio between its height above the horizontal plane (Y) in atlas and patients' brains as employed, for example, by Riechert and Hassler (1955), cannot constitute a correction factor valid for every case.

For the lateral coordinate, a correction factor is also required and Riechert and Hassler have made use of the ratio between the thalamic breadths in the atlas and the patients' brains. This dimension is measured in the antero-posterior encephalogram as the horizontal distance between the septum pellucidum and a perpendicular through the upper and outer angle of the lateral ventricle. That this latter line is not a reliable index of the outer border of the thalamus was shown by Talairach et al. who concluded that there was no single "lateral" correction factor valid for all cases. It was suggested that for any one nucleus a value should be employed representing the mean of its coordinates as determined in anatomical studies. The findings of the present investigation are in support of these conclusions.

\section{Limits of Accuracy}

When the best available correction factor has been applied to each of the three coordinates an attempt must be made to answer the question, What are the limits of accuracy of which the stereotactic technique is capable? It is clear that three groups of factors are relevant:-

(1) Distortion during Fixation and Processing.This factor concerns the preparation of the "atlas brain". It should at once be emphasized that the dimensions in vivo of this brain, including "ventricular" and "total thalamic" lengths, cannot be known. The additional unavoidable distortion occurring after death and during fixation and processing has already been discussed.

(2) Individual Variation.--Reasons for the rejection of the posterior commissure as a reference point for antero-posterior measurements in the thalamus have been presented. Even if use is made, as suggested, of the relative constancy of the position of nuclei along the total thalamic length, it is believed that accuracy cannot be better than \pm 0.75 $\mathrm{mm}$. in the zero horizontal plane and will be poorer for points above or below it.

In the vertical direction, however, the problem of variation would appear to be insuperable and at least as far as the thalamus is concerned vertical accuracy will range from \pm 2.5 to $3.5 \mathrm{~mm}$. according to the antero-posterior level.

The appearances of the globus pallidus at the 100 , 75 , and $50 \%$ thalamic levels show little variation, suggesting that its antero-posterior disposition may parallel that of the thalamus. In the vertical and lateral directions variation is considerable and would appear to be random.

(3) Instrumental.- The use of radiographic control as, for example, by Hassler and Riechert (1954) and Leksell (1949), ensures that the position of the needle tip shall be exactly that calculated from the stereotactic atlas after application of the appropriate correction factors. In this way instrumental error can be reduced to negligible proportions.

It will thus be apparent that the accuracy of the stereotactic technique in the human brain will be determined not by the mechanical precision of a particular instrument but by the unavoidable shortcomings of every atlas and by our limited ability to assess and to correct for the major factor of individual variation. It is to be emphasized that studies of the latter should be based not only on an adequate number of specimens but also upon histological investigations which alone can reliably define the boundaries of diencephalic nuclei.

In conclusion, a coordinate system based upon a horizontal plane through the posterior commissure has been shown to be subject to the variability of the position of the latter (in antero-posterior and vertical directions). Nevertheless it seems unlikely that any more satisfactory plane can be defined for the diencephalon on the basis of structures identifiable in an encephalogram of the third ventricle. The present writers are therefore of the opinion that the future of the stereotactic technique probably lies in the direction of the development of physiological methods which will permit the exploration of nuclei and fibre systems with minimal trauma until the target is reached as judged by physiological criteria. The role of the three-coordinate system (using optimal corrections for individual variation) would then be to define the minimal volume of tissue to be explored.*

\footnotetext{
* The authors regret that they have been unable to gain access to a copy of "Introduction to Stereotaxis with an Atlas of the Human Brain" by G. Schaltenbrand and P. Bailey (1958).
} 


\section{SUMMARY}

Wide individual variation in the antero-posterior and vertical position of the posterior commissure was demonstrated in a histological investigation of 40 hemispheres.

The posterior commissure was found to bear no constant relationship to the thalamic nuclei in the antero-posterior or vertical plane.

The antero-posterior range of the position of the posterior commissure was from 26 to $41 \%$ of the total thalamic length. This difference represented $5.3 \mathrm{~mm}$. in an average total thalamic length of $34.5 \mathrm{~mm}$.

In the vertical plane the position of the posterior commissure varied by up to $6.5 \mathrm{~mm}$. with respect to the upper surface of the thalamus.

A proportionate arrangement of the thalamic nuclei in the antero-posterior axis was demonstrated in all 53 hemispheres.

A less inexact method was suggested for the determination of the antero-posterior coordinate of a thalamic nucleus. No comparable method appeared feasible for the vertical coordinate.

Considerable variation was demonstrated in the lateral and vertical coordinates of the globus pallidus.

Limitations of accuracy in human stereotactic brain surgery were discussed, the paramount importance of individual variation being stressed.

We are indebted to Dr. R. D. Hoare, consultant neuroradiologist to the Guy's-Maudsley Neurosurgical Unit, for permission to make use of the series of encephalograms.

\section{REFERENCES}

Brierley, J. B., and Beck, E. (1957). Proc. 1st Int. Congr. neurol. Sciences. (6th Int. Congr. Neurol.) Brussels, Excerpta Med. (Amst.), 38-39, Sect. VIII, 70, Suppl. (Congress issue).

Delmas, A., and Pertuiset, B. (1954). Presse méd., 62, 458.

Hassler, R., and Riechert, T. (1954). Nervenartz., 25, 441.

Horsley, V., and Clarke, R. H. (1908). Brain, 31, 45.

Leksell, L. (1949). Acta chir. scand., 99, 229.

Loewenfeld, I. E., and Altman, R. (1956). J. Neuropath. exp. Neurol., $15,181$.

Mark, V. H., and Yakovlev, P. I. (1955). Anat. Rec., 121, 745.

Olszewski, J.' (1951). Ibid., 109, 332.

Riechert, T., and Hassler, R. (1955). Personal communication.

Spiegel, E. A., and Wycis, H. T. (1952). Stereoencephalotomy (Thalamotomy and Related Procedures). Part I. Methods and Stereotaxic Atlas of the Human Brain, p. 38. Grune and Stratton, New York.

- Marks, M. and Lee, A. J. (1947). Science, $106,349$.

Talairach, J., Ajuriaguerra, J. de, and David, M. (1952). Presse méd. 60,605.

—, David, M. Tournoux, P., Corredor, H., and Kvasina, T. (1957) Atlas d'Anatomie Stéréotaxique. Masson, Paris. 